\title{
Treatment of periprosthetic supracondylar femoral fractures using the Ilizarov method (report of two cases)
}

\author{
Yılmaz Tomak*, Yılmaz Şahin ${ }^{\mathrm{b} *}$, Bülent Diri ${ }^{\mathrm{b}}$ \\ ${ }^{a}$ Department of Orthopaedics and Traumatology, Faculty of Medicine, Ondokuz Mayis University, Samsun, Turkey \\ ${ }^{b}$ Department of Orthopaedics and Trauma Surgery, Medicana International Samsun Hospital, Samsun, Turkey
}

\section{ARTICLE INFO}

\section{Article History}

Received

$11 / 04 / 2012$

Accepted

$06 / 06 / 2012$

\section{* Correspondence to:}

Yilmaz Şahin

Department of Orthopaedics and Trauma

Surgery, Medicana International Samsun

Hospital, Canik, Samsun, Turkey

e-mail: yilmazfsahin@hotmail.com

\section{ABSTRACT}

Periprosthetic fractures are technically challenging problems for orthopaedic surgery, as they require the skills of reconstruction surgery as well as those of revision arthroplasty. Periprosthetic fractures in patients with total hip arthroplasty (THA) were reported to occur at a rate of $0.1 \%$. The same ratio for patients with total knee arthroplasty is $0.3-$ $5.5 \%$. The patients with periprosthetic fractures have several medical conditions such as osteoporosis, neuropathy, rheumatoid arthritis. Non-operative treatment of periprosthetic femoral fractures in patients with total hip arthroplasty have high complication rates, and is not therefore choiced unless medical comorbidities preclude surgery. Internal fixation methods are the first choice for periprosthetic femoral fractures, it is not possible to apply of these methods in some conditions such as infection, poor quality of bone, the patients with general comorbidities and some configurations of the fracture. In this paper, periprosthetic femoral supracondylar fractures treated with Ilizarov method was presented in 2 cases.

J. Exp. Clin. Med., 2013; 30:69-73

\section{Keywords:}

Arthroplasty

Femoral fracture

Femur

Ilizarov method

Periprosthetic fracture

\section{Introduction}

Periprosthetic fractures in patients with total hip arthroplasty were reported to occur at a rate of $0.1 \%$ (Petty, 1991). The same ratio for patients with total knee arthroplasty is 0.3 $5.5 \%$ (Younger et al.,1998). In the presence of a stable total hip arthroplasty, fractures on the distal of the femoral stem are typically managed with internal fixation methods such as dynamic condylar screw plates (DCP), Condylar plates, May plates, minimal invasive plate systems with or without cables and strut allografts (Fulkerson et al., 2007). Use of these plates and allografts usually causes significant periosteal stripping because of extensile surgical approaches. This kind of the surgery has more complication rates. For decreasing complications, it has been recommended minimal invasive fixation methods which allow minimal disruption of periosteal blood supply and less blood loss. Abhaykumar and Elliott, (2000) have reported good results in a series of seven patients treated by percutaneous fixation of periprosthetic fractures with DCP. Locking plates are applicated on the bone without friction between the bone and plate. The LISS (less invasive skeletal stabilisation, Synthes, Paoli, PA) plate is particularly used in patients with poor quality of soft tissue (Fulkerson et al., 2007). However, internal fixation methods are the first choice for periprosthetic femoral fractures, it is not possible to apply these methods in some conditions such as infection, poor quality of bone, the patients with general comorbidities and some configurations of the fracture. The Ilizarov method is an important alternative when internal fixation methods can not be used.

We reported two cases of a 83-year-old man with total hip arthroplasty and a 74-year-old woman with partial hip arthroplasty in whom periprosthetic femoral supracondylar fractures were treated with closed reduction and external fixation by the Ilizarov method.

\section{Case 1}

A 83-year-old man who was applied multiple revision arthroplasties was sustained periprosthetic fracture of the right distal femur. On plain radiographs, right supracondylar femur showed a displaced comminuted fracture distal to the stem, 
which was classified as type $\mathrm{C}$ according to the Vancouver classification (Ninan et al., 2007) (Fig. 1a, b). The patient had been treated with right total hip arthroplasty due to osteoarthritis secondary to avascular necrosis eighteen years ago. Two months after the first operation, revision procedures had been applied in several years. Custom made type femoral prosthesis had been applied at the last revision procedure because of severe bone defects of the proximal femur.

He had bad health conditons with congestive heart failure and type II diabetes mellitus. We thought that an operation procedure could applied with significant blood loss, a long time by extensive anaesthesia, high morbidity and mortality risks. For this reason, we decided on closed reduction and external fixation of the periprosthetic fracture of the right femur, using an Ilizarov circular external fixator. The frame was preassembled before surgery three carbon rings were used for fixation and the whole frame was connected with threaded rods. The first wire was passed approximately $2.0 \mathrm{~cm}$ below the distal tip of the femoral stem, perpendicular to the anatomical axis of the femur. This wire was connected to the proximal ring. Another wire and a $5.0 \mathrm{~mm}$ half pin were also connected to the proximal ring after application procedure. Then, the closed distraction was applied to the leg manually. While the position of the fracture fragments was controlled with image intensifier, the wires and half pins were passed to the femoral condylar region and they were connected the middle ring on the femoral condyles. A transverse wire and a $5.0 \mathrm{~mm}$ half pin were passed the proximal tibia, and connected to the distal ring. The patient was mobilized with crutches without weight-bearing immediately after surgery. It was corrected acutely with translation construct using original Ilizarov parts. The most distal ring and its wire and half pin were removed postoperatively after one and half month. In this way, range of motion exercises of the knee was started. In addition, it was permitted partial weight-bearing at this time. When it was seen adequate callus formation on the radiographs, it was permitted full weight-bearing at the postoperative three months (Fig. 1c, d). Stable bone fusion was obtained at the postoperative six months, and the frame was removed in outpatient clinic. At the latest follow-up, 24 months after frame removal, the patient had returned to a normal life without pain and required a simple cane (Fig. 1e, f).
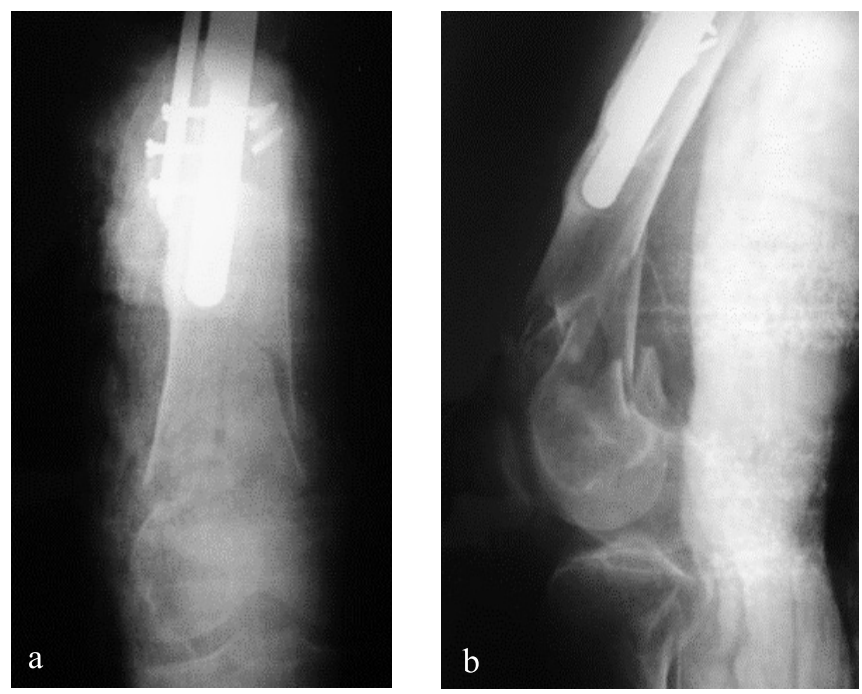

Fig. 1a, b. Case 1. Initial anteroposterior (a) and lateral (b) radiographs of the left distal femur below a femoral stem.
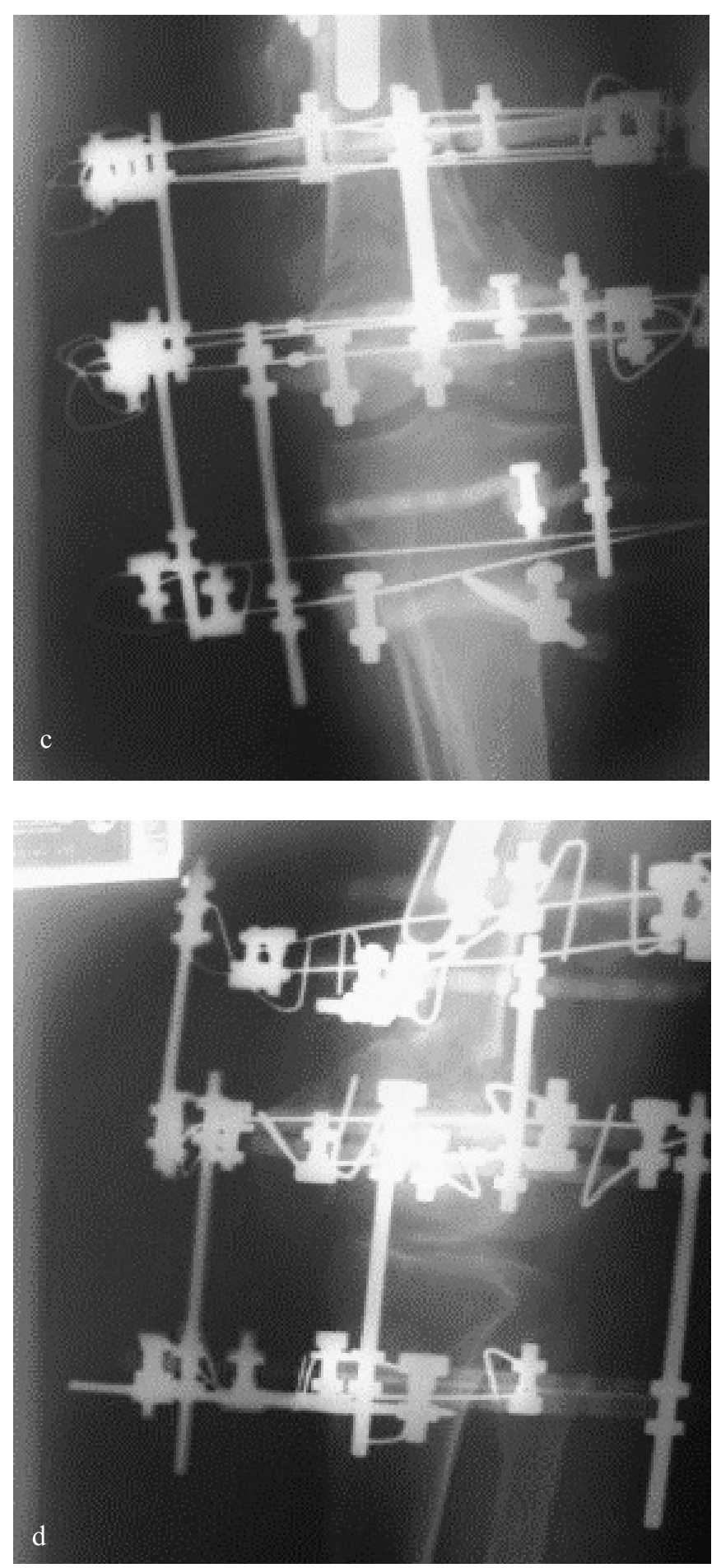

Fig. 1c, d. Postoperative anteroposterior (c) and lateral (d) radiographs after application of the frame.

\section{Case 2}

A 74-year-old woman was sustained periprosthetic fracture of the left distal femur due to falling in the house. On plain radiographs, left supracondylar femur showed a displaced transverse fracture distal to the stem, which was classified as type $\mathrm{C}$ according to the Vancouver classification (Ninan et al., 2007) (Fig. 2a, b). The patient had been treated with right partial hip arthroplasty due to femoral neck fracture three years ago. She had chronic obstructive lung disease, hypertension, type II diabetes mellitus and osteoporosis. We aimed an operation that needed a short time period with negligible blood loss and stable fixation. Therefore, we chose closed reduction and external fixation with the Ilizarov method. The frame was preassembled before surgery. 

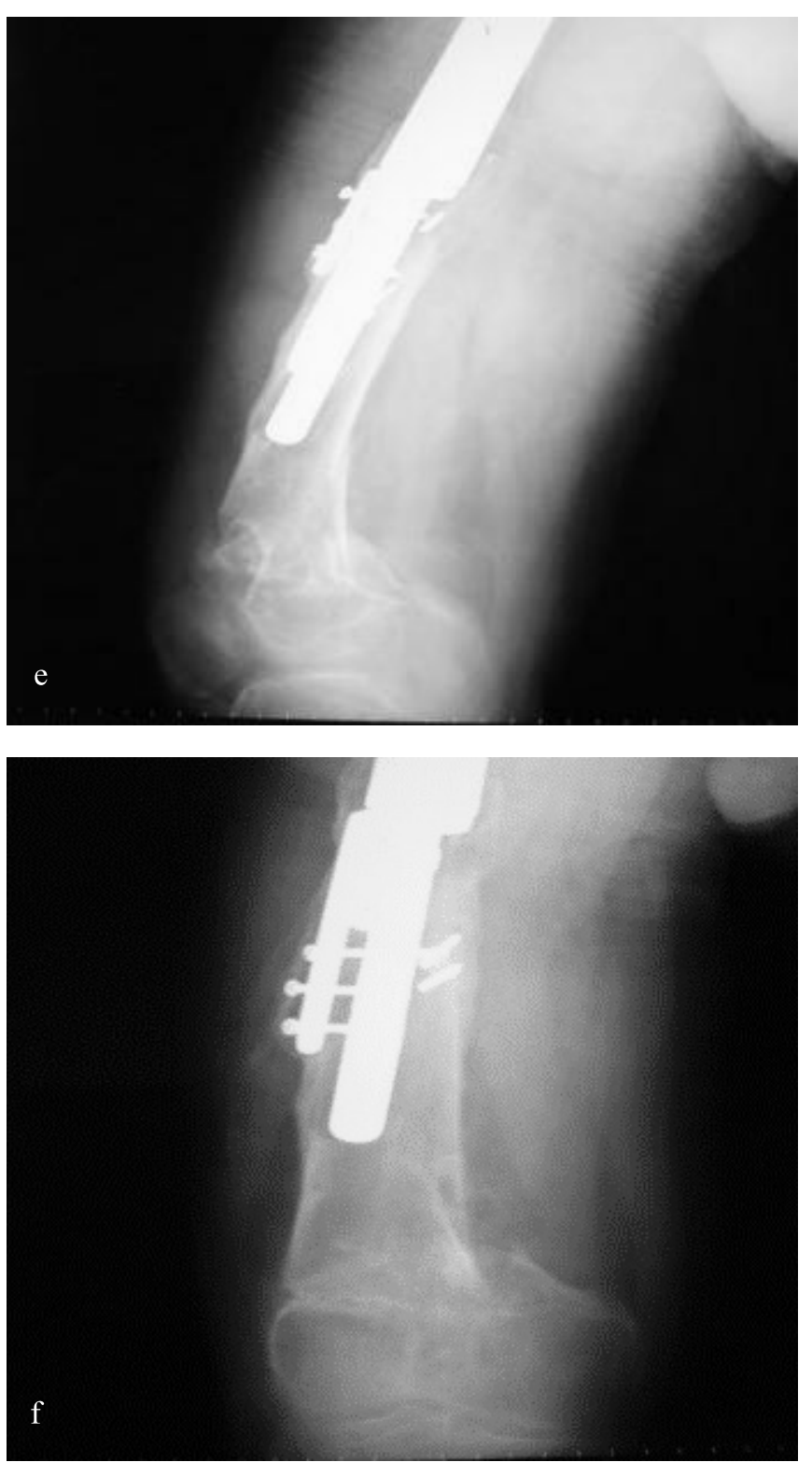

Fig. 1e, f. Lastest follow-up anteroposterior (e) and lateral (f) radiographs showing successful fracture union at 24 months.

Three carbon rings were used for fixation and the whole frame was connected with threaded rods. The proximal and middle carbon rings fixed the fracture of the distal femur below femoral stem and the distal carbon ring was applied on the proximal tibia for securing fracture fixation. The first wire was passed approximately $5.0 \mathrm{~cm}$ below the distal tip of the femoral stem, perpendicular to the anatomical axis of the femur. After this, the surgical procedure was exactly the same as the first event. The most distal ring and its wire and half pin were removed postoperatively after one and a half month. In this way, range of motion exercises of the knee was started. In addition, it was permitted partial weight-bearing at this time. When it was seen adequate callus formation on the radiographs, it was permitted full weight-bearing at the postoperative three months (Fig. 2c). Stable bone fusion was obtained at the postoperative five months, and the frame was removed in outpatient clinic. At the latest follow-up, 18 months after frame removal, the patient had returned to a normal life without pain and did not require a walking aid (Fig. 2d, e).
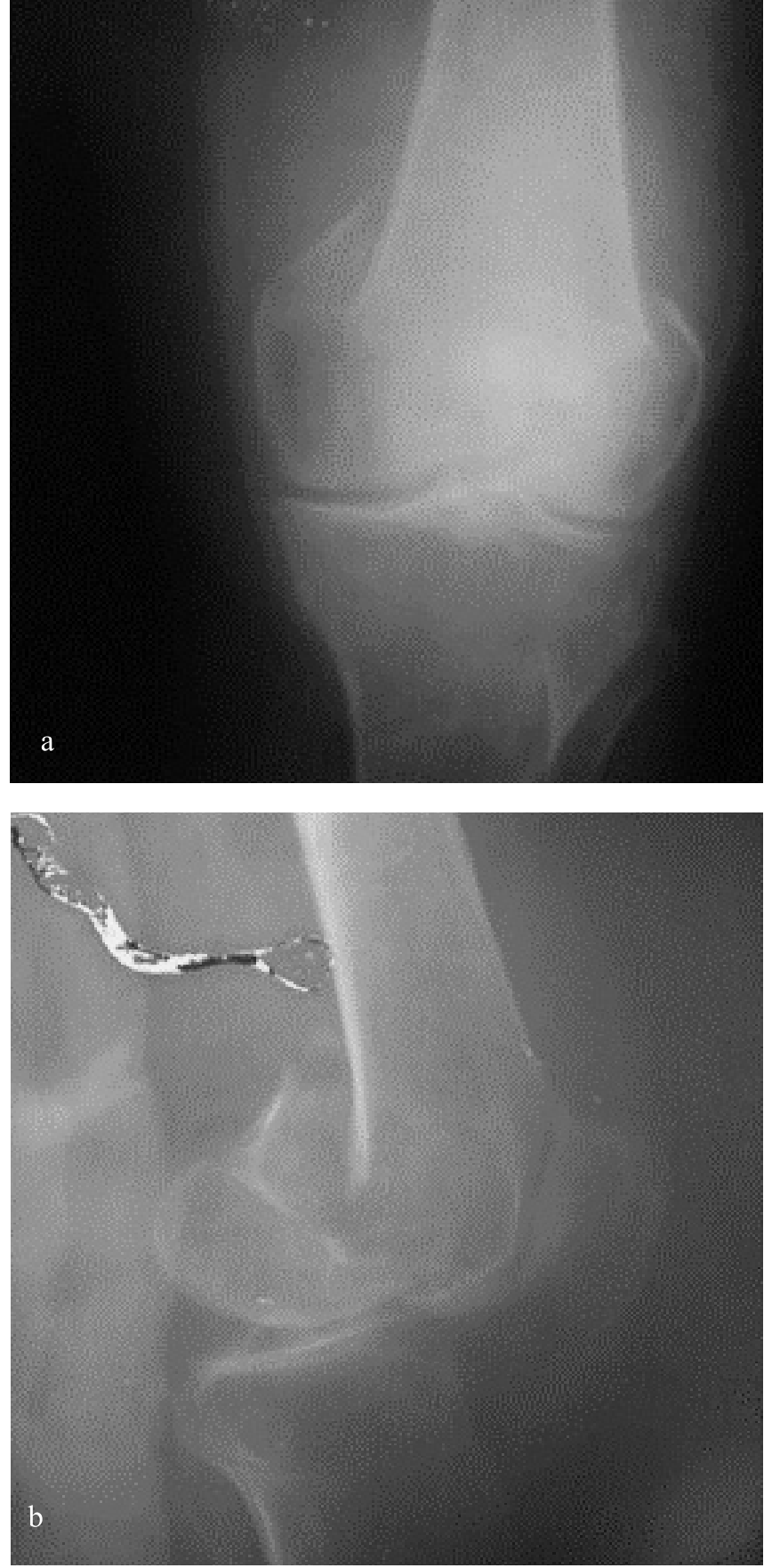

Fig. 2a, b. Case 2. Initial anteroposterior (a) and lateral (b) radiographs of the left distal femur below a femoral stem.

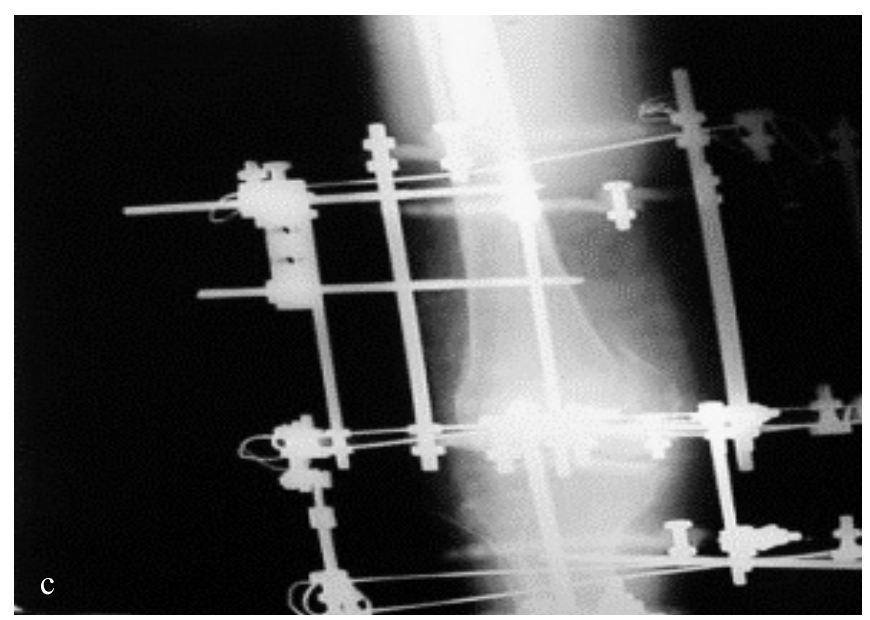

Fig. 2c. Postoperative anteroposterior (c) radiographs after application of the frame. 

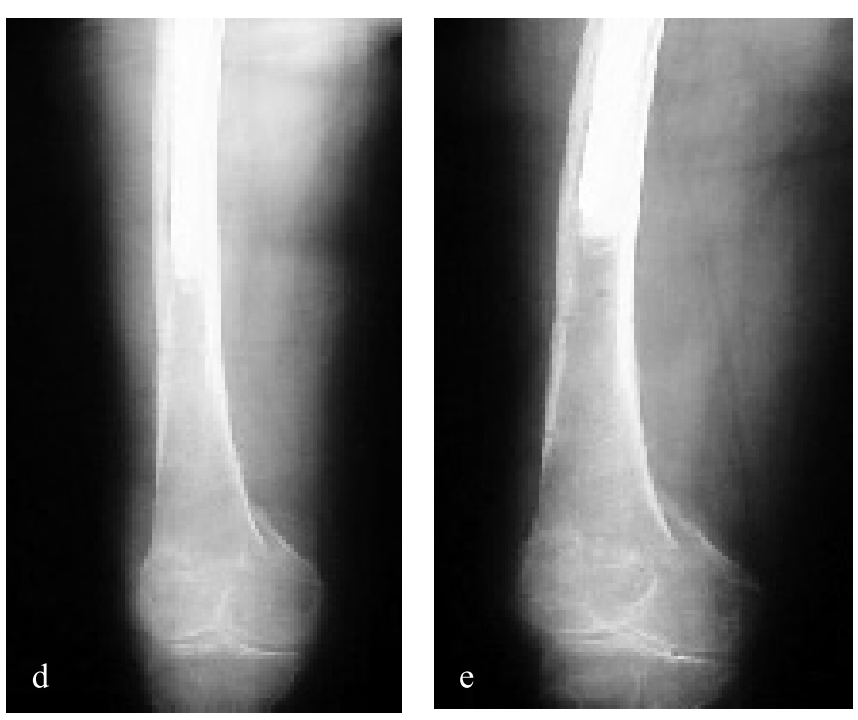

Fig. 2d, e. Lastest follow-up anteroposterior (d) and lateral (e) radiographs showing successful fracture union at 18 months.

\section{Discussion}

Periprosthetic femoral fractures are seen with increasing rates in connection with increase in the number of total hip arthroplasties performed. Late fractures have been reported to be less than $1 \%$ after primary arthroplasty and up to $4 \%$ following revision surgery. The incidence of intraoperative fractures is higher than late fractures but it can be solved by several alternatives such as changing stem size and length, applying many fixation materials without difficulties about surgical granulation tissues.

The treatment of periprosthetic femoral fractures can be changed by various factors including fracture configuration, bone quality, soft tissue quality, mental and physical status of the patient and prosthesis design. Internal fixation devices and methods are the unique choice for periprosthetic fractures around a femoral stem. It is stated that conventional plating with or without cerclage wiring involved extensive dissection and potentially compromises periosteal supply (Beals and Tower, 1996). Minimally invasive insertion of some fixation devices such as DCP, LISS, cloverleaf plate, limited contactdynamic compression plate is an advantageous approach due to minimal disruption of periosteal blood supply.

The aim of the treatment of fractures of elderly patients is immediate reestablishment of their functional status. It necessitates a safe fracture fixation and an adequate rehabilitation for the successful result. Long term immobilization of these patients due to failed surgical or conservative treatment methods may cause thromboembolic problems, decubitus ulcers and cardiopulmonary insufficiencies. The consequence is high morbidity and mortality rates.

Fracture healing of patients with osteoporosis is poor.
Blood circulation of the bone and soft tissues must be preserved as well as possible. Ilizarov method is relatively atraumatic and periosteal circulation is not disturbed (Belhan et al., 2008). In addition, it must respect to principles of biological fracture fixation. Because of the decreased gripping power of plate and screw fixation in osteoporotic bone fractures, these kind of methods can have a high failure rate, ranging from $10 \%$ to $25 \%$ (Cornell, 2003). It has been reported bad result as high as $25 \%$ of the elderly patients with supracondylar femoral fractures treated with angled blade plate (Beals and Tower, 1996; Younger, 1998).

In two cases, we used Ilizarov external fixator successfully. The Ilizarov method for a periprosthetic femoral fracture below a total hip arthroplasty has been reported by Sakai et al. (2007). They had initially applied open reduction and internal fixation for the left periprosthetic femoral fracture using a supracondylar plate system. Thereafter, they diagnosed a deep infection around the femoral fracture and removed the supracondylar plate system and applied Ilizarov external fixator for the fracture. The Ilizarov method has four advantages used for a periprosthetic femoral fracture after total hip arthroplasty (THA). First, percutaneous multiple transverse wires can be inserted in various directions and at various levels. Second, deformity at the fracture site can be corrected 3-dimensionally using hinge parts. In the present study, a valgus deformity was corrected, although displacement remained. Third, there is little risk of surgical damage or transfusion. And fourth, early full-weight bearing leads to callus formation by micromotion of the fracture site (Sakai et al., 2007). They choiced the Ilizarov method because of a deep infection which has developed open reduction internal fixation using a supracondylar plate system. Whereas, we preferred the Ilizarov method for two cases with periprosthetic supracondylar femoral fractures as the first choice. Besides, there was no infection finding for two cases.

For the Ilizarov method, main fear is transformation from pin tract infection to prosthesis infection. Periprosthetic femoral fractures are challenging cases and demanding to treat. The Ilizarov method provides a superior alternative for the surgical management of these fractures with poor local and general factors. It should be especially considered when classical treatment methods have important disadvantages. We prefered the Ilizarov method for two cases. Because, they have additional medical problems, poor bone quality and high anaesthesia risks. The rate of success of internal fixation with osteoporotic bone fractures is lower than the Ilizarov method. Mechanical failures are higher with internal fixation methods. Whereas, the Ilizarov method is more advantageous due to multidirectional wire and half pin fixations for osteoporotic bone fractures.

\section{REFERENCES}

Abhaykumar, S., Elliott, D.S., 2000. Percutaneous plate fixation for periprosthetic femoral fractures a preliminary report. Injury. 31, 627-630.

Beals, R.K., Tower, S.S., 1996. Periprosthetic fractures of the femur. An analysis of 93 fractures. Clin. Orthop. Relat. Res. 327, $238-246$.

Belhan, O., Ekinci, A., Karakurt, L., Y1lmaz, E., Serin, E., 2008. The treatment of femoral shaft fractures in adults with hybrid Ilizarov external fixator. J. Dis. Relat. Surg. 19, 50-54.

Cornell, C.N., 2003. Internal fracture fixation in patients with osteoporosis. J. Am. Acad. Orthop. Surg. 11, 109-119.

Fulkerson, E., Tejwani, N., Stuchin, S., Egol, K., 2007. Management of periprosthetic femur fractures with a first generation locking plate. Injury. 38, 965-972.

Ninan, T.M., Costa, M.L., Krikler, S.J., 2007. Classification of femoral periprosthetic fractures. Injury. 38, 661-668.

Sakai, T., Ohzono, K., Nakase, T., Lee, S.B., Manaka, T., Nishihara, S., 2007. Treatment of periprosthetic femoral fracture after cementless total 
hip arthroplasty with ilizarov external fixation. J. Arthroplasty. 22, 617-620.

Petty, W., 1991. Total hip arthroplasty: Complications. In: Petty, W., editor. Total joint replacement. Philadelphia: WB Saunders. 287-314.

Younger, A.S., Dunwoody, I., Duncan, C.P., 1998. Periprosthetic hip and knee fractures: The scope of the problem. Instr. Course Lect. 47, 251256. 